\title{
Comment soutenir les finances publiques face aux catastrophes naturelles
}

Écrit par : Catherine Gamper, Direction de la Gouvernance publique de l'OCDE

Dernière mise à jour : 30 octobre 2019

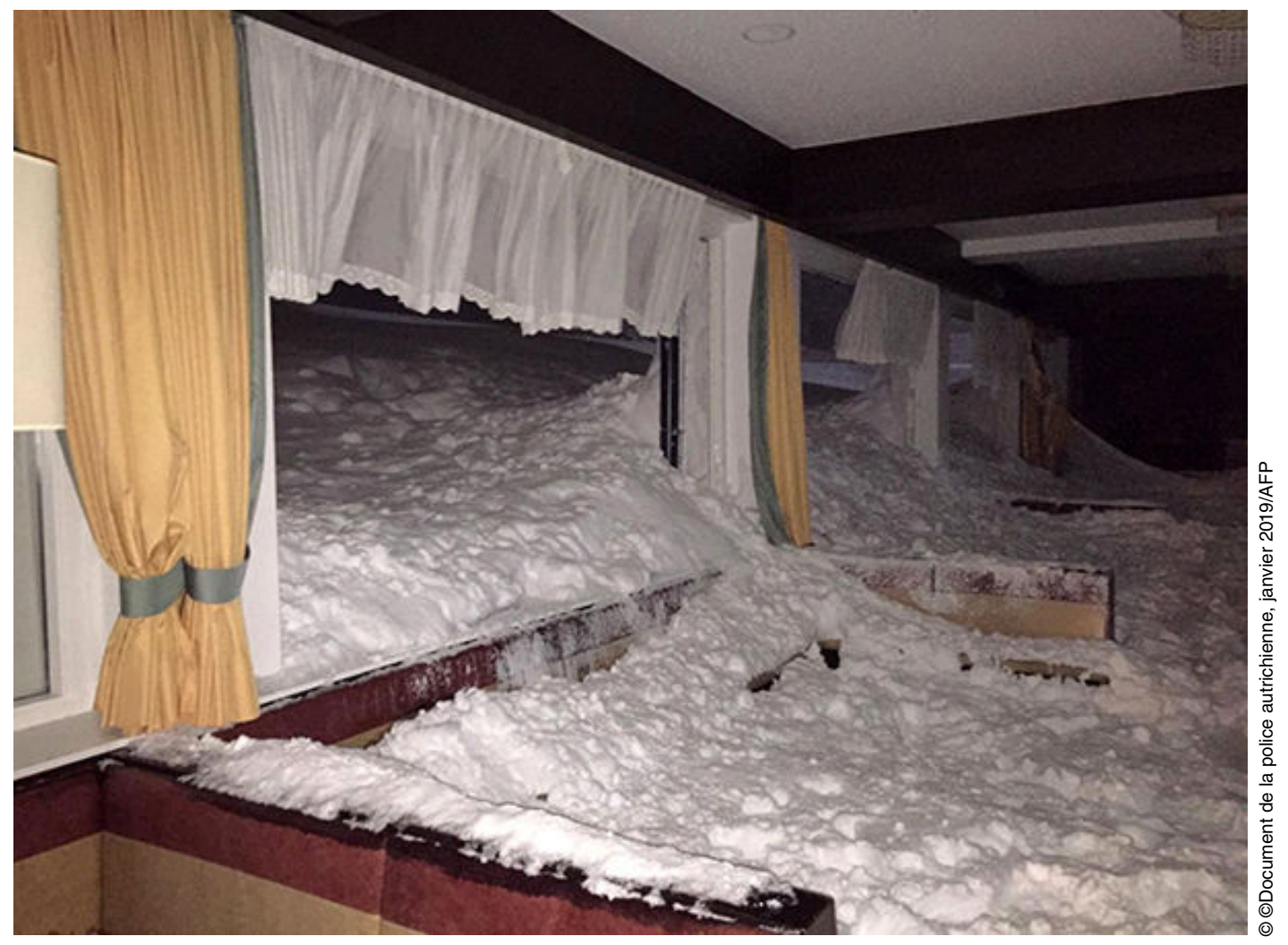

Les catastrophes naturelles arrivent en général sans s'annoncer, c'est pourquoi les autorités publiques doivent être bien préparées, y compris financièrement. Que peuvent-elles faire pour éviter les surprises et renforcer leur résilience budgétaire?

Un matin de 1988, emmitouflée dans mes vêtements d'hiver, je me préparais à braver de nouveau le trajet qu'il me fallait parcourir dans le froid et l'obscurité jusqu'à l'école, lorsque le téléphone a sonné. Ma mère nous a annoncé une bonne nouvelle inattendue : pas d'école aujourd'hui. Mais nous avons rapidement compris qu'il n'y avait pas lieu de se réjouir. Si nous ne pouvions aller à l'école, c'est parce qu'une énorme avalanche avait englouti la moitié de notre village. Certains de mes camarades étaient indemnes et quelques-uns s'étaient réveillés 
au contact de la neige tombant sur leur lit, mais d'autres avaient malheureusement perdu des proches.

Ayant grandi en Autriche, un petit pays entouré de montagnes enneigées et doté de nombreuses étendues d'eau, j'ai toujours eu pleinement conscience des risques que faisaient peser les événements climatiques sur le pays. En effet, $13 \%$ des Autrichiens sont menacés par les avalanches, les débordements de torrents ou les inondations, et le changement climatique devrait encore accroître l'exposition de l'Autriche à ces risques.

Si le pays a pris des mesures de longue date face à ces conditions climatiques défavorables, en investissant dans la réduction des risques de catastrophes, les coûts qui en résultent pour le gouvernement restent élevés. Le montant annuel moyen des pertes, estimé à 210 millions EUR, ne semble peut-être pas si important, mais il inclut des événements extrêmes comme les inondations de 2003 qui ont causé 3 milliards EUR de dégâts environ et ont donné lieu à des indemnisations versées pour l'essentiel par l'État.

L'Autriche n'est pas la seule à faire face à de tels coûts. De nombreux pays membres et non membres de l'OCDE sont confrontés aux effets dévastateurs des catastrophes naturelles sur les finances publiques. Par exemple, le séisme, le tsunami et la catastrophe nucléaire qui ont eu lieu en 2011 dans le Tohoku, au Japon, ont non seulement entraîné la perte tragique de nombreuses vies humaines, mais aussi contribué à une contraction de $2 \%$ de l'économie au cours du trimestre qui a suivi ces événements, et conduit en 2012 à une hausse des dépenses réalisées par le gouvernement central pour la reconstruction, équivalant à $8 \%$ du PIB du Japon et à plus de $20 \%$ du budget général du pays.

L'incidence budgétaire des catastrophes n'est pas seulement liée à leur ampleur. Bien qu'une catastrophe locale unique soit peu susceptible d'affecter la comptabilité nationale, des incidents locaux plus modestes mais fréquents peuvent avoir des incidences budgétaires et économiques considérables, au même titre que les grandes catastrophes.

Quelle que soit l'ampleur de l'incident, ce sont souvent les pouvoirs publics qui prennent en charge l'essentiel des coûts de redressement et de reconstruction. Il peut s'agir de réparer les actifs publics endommagés, d'apporter un secours immédiat aux ménages et de contribuer aux efforts de reconstruction de la population, ou de fournir un soutien aux entreprises pour qu'elles restent en activité. Et tandis que ces mesures font monter les dépenses publiques, les recettes publiques risques de baisser par suite du ralentissement de l'activité économique. Ces effets combinés pèsent lourdement sur le bilan de l'État.

Que peuvent alors faire les pouvoirs publics pour garantir leur résilience budgétaire ; dans un récent rapport intitulé Fiscal Resilience to Natural Disasters, l'OCDE et la Banque mondiale présentent des instructions et des idées pratiques sur la manière dont les gouvernements gèrent les passifs liés aux catastrophes, et 
proposent des moyens de mieux les contrôler et de réduire leurs incidences budgétaires à l'avenir.

\section{How to shore up public finances against the unexpected}

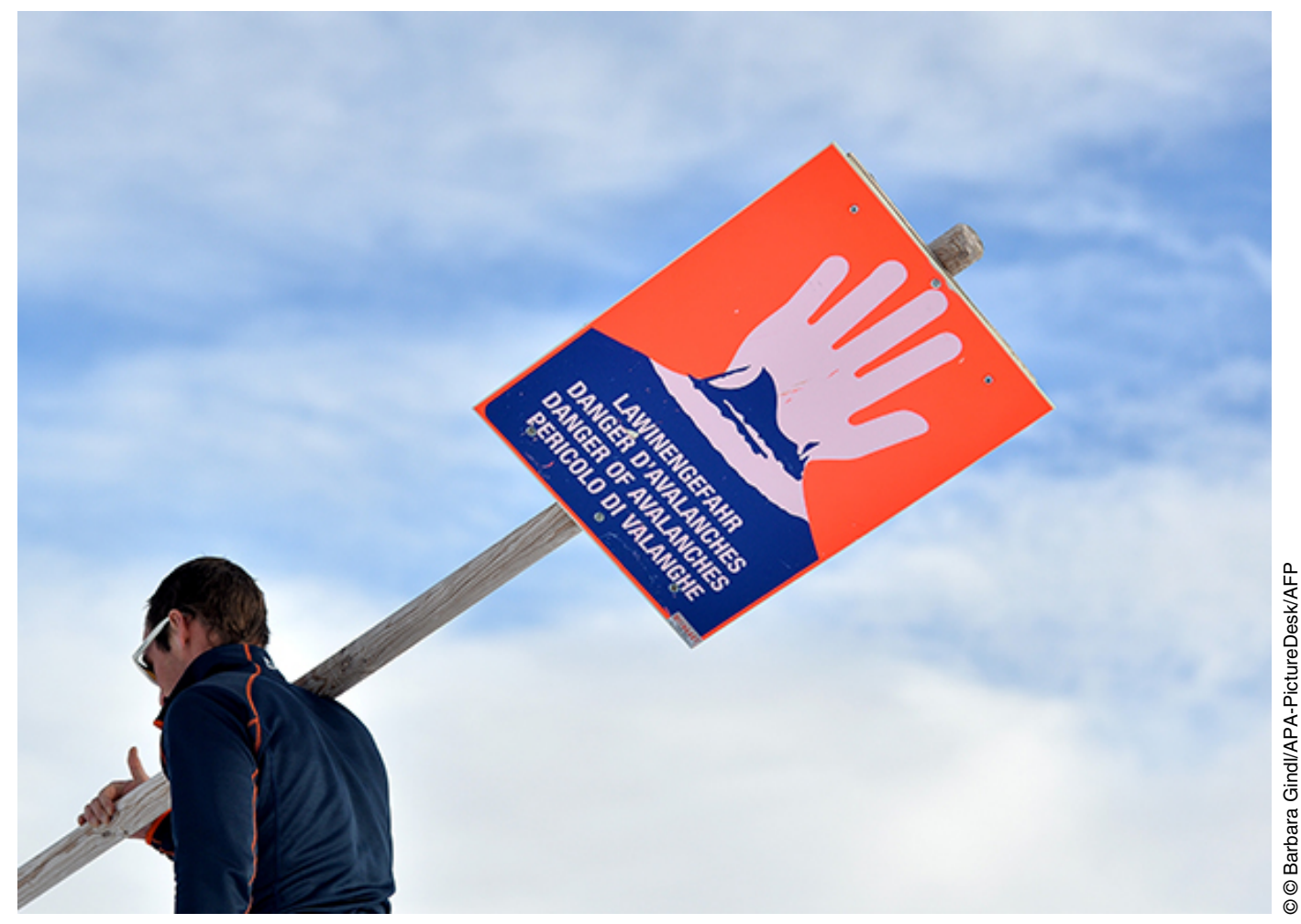

Le rapport constate qu'il est possible, en définissant explicitement les règles applicables aux engagements des pouvoirs publics à verser des indemnités en cas de pertes dues aux catastrophes, de limiter ces passifs, comme c'est le cas au Japon, par exemple. Dans d'autres pays, comme la Colombie, les règles sur l'indemnisation publique, qui restent très générales, renforcent la confiance des populations à l'égard de l'aide financière apportée par l'État et diminuent l'incitation des parties prenantes touchées à agir en amont et à investir dans la protection de leurs propres actifs.

Le rapport fait également observer que les pays devraient être mieux informés de l'ampleur des conséquences que peuvent avoir les catastrophes sur leur budget. Même si beaucoup de pays disposent de nombreuses données, celles-ci ne sont pas toujours compilées de façon à permettre une estimation des effets budgétaires. Les déclarations sur le risque budgétaire pourraient contenir une mention qualitative, par exemple « risques importants, mais éloignés ", de façon à attirer l'attention des décideurs sur la nécessité d'anticiper et de mieux gérer les risques de catastrophes.

Mais les pouvoirs publics ont encore une grande marge de manœuvre, notamment pour contrôler plus étroitement les passifs en lien avec les 
catastrophes, par exemple en améliorant les conditions auxquelles est accordé le soutien financier à la reconstruction.

Prenons un autre exemple autrichien : à la suite d'un très grave glissement de terrain en 2015, le maire de la localité touchée a fièrement annoncé que « les familles qui ont perdu leur maison ont décidé de ne pas partir. Et nous les aiderons à reconstruire leur logement exactement là où il se trouvait ».

Si le lieu de vie, la maison et la communauté présentent de toute évidence une grande importance pour la population, cette anecdote montre pourquoi les passifs continuent d'augmenter. La réduction des risques, et en particulier le souci de « mieux reconstruire » devrait être au cœur des mesures de réduction des passifs. Il importe également de former les entreprises et les ménages aux risques de catastrophe et au devoir qu'ils ont de renforcer leur propre résilience. Et, pour ne pas avoir à payer de façon répétée pour les mêmes sinistres évitables, les pouvoirs publics devraient envisager des incitations qui pourraient consister à abaisser les taux de remboursement si les demandes d'indemnisation se répètent.

Les catastrophes et les événements extrêmes semblent plus nombreux aujourd'hui que lorsque j'étais enfant et risquent encore de s'accentuer, notamment en raison du changement climatique. Les pouvoirs publics devront s'efforcer de définir des règles claires en matière d'assistance et d'indemnisation des sinistres, afin d'éviter que les passifs liés aux catastrophes naturelles n'entraînent aussi des catastrophes financières.

COObservateur de l'OCDE n³19, octobre 2019

\section{Références}

OCDE-Banque mondiale (2019), Fiscal Resilience to Natural Disasters: Lessons from Country Experiences, Éditions OCDE, Paris, voir OECD iLibrary ou http:// dx.doi.org/https://doi.org/10.1787/27a4198a-en

OCDE (2018), Assessing the Real Cost of Disasters: The Need for Better Evidence, OECD Reviews of Risk Management Policies, Éditions OCDE, Paris, voir OECD iLibrary http://dx.doi.org/https://doi.org/10.1787/9789264298798-en

OCDE (2017), Boosting Disaster Prevention through Innovative Risk Governance: Insights from Austria, France and Switzerland, OECD Reviews of Risk Management Policies, Éditions OCDE, Paris, voir OECD iLibrary ou http:// dx.doi.org/https://doi.org/10.1787/9789264281370-en. 